\title{
Osteomyelitis in Children with Sickle Cell Disease: A Challenging Diagnosis: Case Report from Cameroon
}

\author{
Djike Puepi Fokam Yolande ${ }^{1,2^{*}}$, Kukwah Anthony Tufong2,3, Tagakou Mboula Jules ${ }^{2,4}$, \\ Andang Paul Mayah', Eposse Ekoube Charlotte ${ }^{6,7}$, Diomede Noukeu Njinkui ${ }^{8}$, \\ Dominique Enyama ${ }^{8}$, Helene Kamo Selangai ${ }^{9}$, Verla Vincent Siysi ${ }^{1,2}$
}

\footnotetext{
${ }^{1}$ Department of Internal Medicine and Paediatrics, Faculty of Health Science, University of Buea, Buea, Cameroon

${ }^{2}$ Buea Regional Hospital, Buea, South West, Cameroon

${ }^{3}$ Department of Microbiology and Parasitology, Faculty of Sciences, University of Buea, Buea, Cameroon

${ }^{4}$ Department of Clinical Sciences, Faculty of Health Sciences, University of Bamenda, Bamenda, Cameroon

${ }^{5}$ Faculty of Health Sciences, University of Buea, Buea, Cameroon

${ }^{6}$ Faculty of Medicine and Pharmaceutical Sciences, University of Douala, Douala, Cameroon

${ }^{7}$ Treatment Centre of Sickle cell Disease, Laquintinie Hospital, Douala, Cameroon

${ }^{8}$ Faculty of Medicine and Pharmaceutical Sciences, University of Dschang, Dschang, Cameroon

${ }^{9}$ Faculty of Medicine and Biomedical sciences, University of Ngaoundere, Ngaoundere, Cameroon

Email: *yolandep2000@yahoo.fr
}

How to cite this paper: Yolande, D.P.F., Tufong, K.A., Jules, T.M., Mayah, A.P., Charlotte, E.E., Njinkui, D.N., Enyama, D., Selangai, H.K. and Siysi, V.V. (2021) Osteomyelitis in Children with Sickle Cell Disease: A Challenging Diagnosis: Case Report from Cameroon. Open Journal of Pediatrics, 11, 208-214.

https://doi.org/10.4236/ojped.2021.112020

Received: March 18, 2021

Accepted: May 28, 2021

Published: May 31, 2021

Copyright $\odot 2021$ by author(s) and Scientific Research Publishing Inc. This work is licensed under the Creative Commons Attribution International License (CC BY 4.0).

http://creativecommons.org/licenses/by/4.0/

\section{cC) (i) Open Access}

\begin{abstract}
Introduction: Sickle Cell Disease (SCD) is the most prevalent genetic disease in the world predominantly in the African population with Sickle Cell Anaemia (SCA) being its dominant form. One of the most frequent complications of SCD is osteomyelitis. SCA is due to a point mutation in the beta globin chain of haemoglobin. This is responsible for the sickled shape of RBCs under low oxygen tension conditions leading to obstruction in the microcirculation. This leads to vaso-occlusive crises (VOC) which has a similar clinical presentation to that of osteomyelitis, another complication of SCD. Case Presentation: We present the case of a three-year-old girl with SCA who presented with an inability to bear weight in a febrile context. A diagnosis of VOC was initially made, which was later on changed to both a left chronic tibial and right distal femoral osteomyelitis following a series of biological, and imaging investigations. Surgical debridement and drainage were performed, resulting 9 weeks later in the involution of fever and leg pain. Conclusion: Osteomyelitis when associated with SCD is a dreadful and deathly disease in low income countries as it also presents like VOC therefore higher suspicion index is recommended. It is therefore important to take this into consideration at an early stage in patients with homozygous sickle cell disease so as to rapidly
\end{abstract}


initiate multidisciplinary care. Appropriate investigations, appropriate antibiotic therapy, and timely surgical intervention would help to greatly reduce morbidity and mortality.

\section{Keywords}

Chronic Osteomyelitis, Sickle Cell Disease, Child

\section{Introduction}

Sickle Cell Disease (SCD) is the most prevalent genetic disease in the world with a higher predominance in populations of African descent. This haemoglobinopathy is a major public health problem in Africa and particularly in Cameroon, where between $20 \%$ to $25 \%$ of the population carries the sickle cell trait [1]. Sickle Cell Anaemia (SCA) is the most severe form of SCD and it is common in geographical areas where malaria is widespread [2]. In SCD, haemoglobin precipitates as insoluble crystals which lead to an abnormal shape and size of Red Blood Cells (RBCs) with subsequent phagocytosis of the affected corpuscles. Under low oxygen concentration, RBCs that contain an abnormal form of Haemoglobin $(\mathrm{Hb})$ become deformed (or sickle-shaped) and rigid. This impedes their ability to pass through microcirculation, with frequent clotting and thrombosis. The consequence of the obstruction is the production of ischemia and infarction. Tissue infarctions, generally referred to as vaso-occlusive crisis (VOC) present as pain or swelling. Infarcts can affect several organs of the patient including brain, lungs, bones and spleen, and are responsible for most clinical manifestations. Previous research has shown higher susceptibility of SCA children towards a rare form of osteomyelitis, i.e. salmonella osteomyelitis [3]. Staphylococcus aureus is the pathogen most commonly involved. Medullary bone infarction and necrosis create an apt condition for bacterial growth and spreading. In these patients, osteomyelitis is commonly seen in the tibia, diaphysis of femur and humerus, along with the infection of the vertebrae. The infection is haematogenous in nature due to its delayed onset. Immunodeficiency caused by splenic dysfunction, tissue infarction, and excess iron content leads to an increased risk of osteomyelitis [4]. In SCA, osteomyelitis diagnosis can be a major problem for healthcare providers, where unsuccessful identification of the disease may end up with severe bone damage and progression of infection to life-threatening status. Patients will suffer pain, swelling, fever and increased flow of inflammatory markers (CRP/ESR) in blood serum. This shows parallel characteristics with that of painful bone crises, which makes differentiation with bone infarction a difficult task. Radiographic findings of osteopenia, sclerosis, and periosteal inflammation are seen in both stages of infection and infarction. Therefore, the radiographic features are nonspecific and primarily normal [4] [5]. We report the case of a three-year-old female sickle cell patient with chronic bone pain, which was later diagnosed as chronic osteomyelitis and cared for. 


\section{Case Presentation}

A three-year-old girl, known sickler compliant on daily folic acid supplements and prophylactic amoxicillin antibiotics, and up-to-date on her immunisation history. She was also seen to have adequate childhood development. She was transferred from the paediatric unit to the orthopaedic unit for better management after presenting with an inability to bear weight of 9 weeks duration.

The child's parents reported a history of intermittent left leg pain increasing in intensity in a context of fever with no signs of inflammation. She was managed for a VOC in the paediatric unit with oral cloxacillin $150 \mathrm{mg} / \mathrm{kg}$ thrice daily for 10 days and daily alcoholic dressings done on the leg, which later on subsided the pain. Three weeks later, the pain reoccurred. There was a fever and signs of inflammation. An X-ray of the left leg done on day 21 of progress was unremarkable, blood workup revealed C-Reactive Protein (CRP) at $96 \mathrm{mg} / \mathrm{l}$ and a Full Blood Count (FBC) showed leucocytosis at 21,400 cells/ $\mu$ l. There was also a severe microcytic anaemia with haemoglobin at $7 \mathrm{~g} / \mathrm{dl}$, and severe thrombocytopenia at 19,000 cells/ $\mu \mathrm{L}$. A blood culture was done which was sterile. A urine culture was done which was positive for Escherichia coli sensitive to Ciprofloxacin, Meropenem, Imipenem, Chloramphenicol, Ofloxacin. Ultrasonography of the urinary tract was normal. The patient was placed on parenteral Imipenem 60 $\mathrm{mg} / \mathrm{kg}$ in four doses for ten days combined with continuous alcoholic dressing of the leg. Pain of the leg was thought to be a VOC. The persistence of symptoms prompted a review from a sickle cell specialist who suspected an osteomyelitis and placed the patient on parenteral Lincomycin $30 \mathrm{mg} / \mathrm{kg}$ in two divided doses daily and oral Cefixime $8 \mathrm{mg} / \mathrm{kg}$ bid daily. For pain Tramadol $1 \mathrm{mg} / \mathrm{kg}$ thrice daily was given alongside Paracetamol syrup $60 \mathrm{mg} / \mathrm{kg}$ in four doses. A second $\mathrm{X}$-ray of the affected leg was requested and done on day 28 of evolution coupled with blood cultures to be done after a therapeutic window of 48 hours.

The X-ray was normal, blood culture was negative, and urine culture was positive for Kluyvera sp. sensitive to Imipenem, Meropenem, Doxycycline, Nitrofurantoin and Quinolones. At this point all parenteral antibiotics were stopped and the patient was placed on oral Nitrofurantoin $50 \mathrm{mg}$ daily alongside all other aspects of management (antalgics and alcohol dressing). Fever subsided by the $10^{\text {th }}$ day of treatment, but the pain in the leg, swelling and inability to bear weight persisted and prompted a second orthopaedic review. On orthopaedic examination, signs of inflammation were found on the left proximal leg. Patient was subjected to a third, X-ray of both legs which revealed a sequestrum surrounded by involucrum on the left proximal tibia with periosteal reaction on the distal right femur (Figure 1).

A pre-operative workup was done preceding a sequestrectomy and drainage of the proximal left tibia, and a drain put in place. The result of the pus culture obtained on the second day post-surgery was positive for Salmonella sp. and sensitive to Imipenem, Ceftriaxone, Chloramphenicol, Ofloxacin and Gentamycin. Her treatment was Ceftriaxone $75 \mathrm{mg} / \mathrm{kg} /$ day and Gentamycin $3 \mathrm{mg} / \mathrm{kg} /$ day. 
Results of the bone biopsy histopathologic analysis were in favour of a "subacute osteitis".

On the second day post-op, the patient became paler and more dyspnoeic; FBC revealed a haemoglobin level drop to $6.8 \mathrm{~g} / \mathrm{dl}$. She was thus transfused $350 \mathrm{cc}$ of whole blood.

Control CRP on the sixth day post-op was at $24 \mathrm{mg} / \mathrm{L}$.

Post-op evolution was favourable as the patient was discharged on the $7^{\text {th }}$ day post-op, relayed on oral thiamphenicol $75 \mathrm{mg} / \mathrm{kg}$ in two doses daily for six weeks, oral paracetamol $60 \mathrm{mg} / \mathrm{kg}$ in four divided doses daily to alternate with oral ibuprofen tid daily in case of pains, alongside daily wound dressing.

Patient came for follow, six weeks later; there was no pain or swelling on the right leg, no fever, and she was now able to bear weight. Blood workup showed leucocytosis at 17,100 cells/ $\mu \mathrm{l}$ of lymphocytic and monocytic predominance. There was also a normocytic anaemia with haemoglobin at $8.2 \mathrm{~g} / \mathrm{dl}$ and thrombocytosis at 784,000/ $\mu \mathrm{l}$. CRP was at $<6 \mathrm{mg} / \mathrm{l}$. X-ray of the legs showed signs of bone repair at the lesion sites (Figure 2).

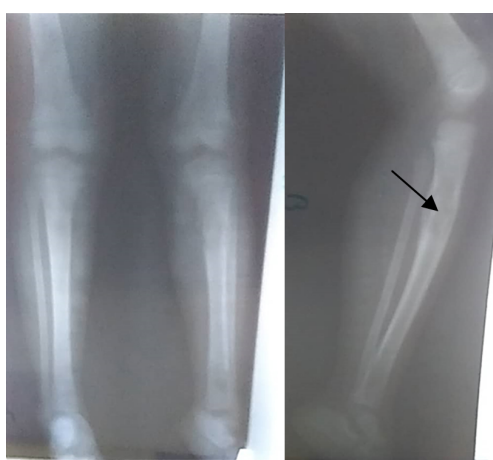

(a)

Figure 1. X-ray of the legs on diagnosis. (a) Anterior view of both legs; (b) lateral view of the left leg.

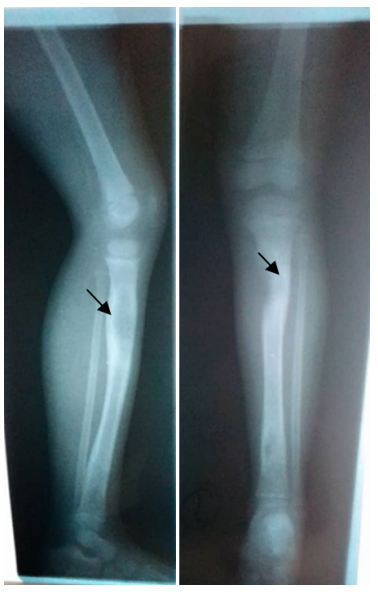

(a)

(b)

Figure 2. X-rays of the legs 6 weeks post-operative. (a) Lateral view of the left leg; (b) anterior view of the left leg. 


\section{Discussion}

We presented a three-year-old, known sickler with a history of chronic long bone pain initially thought to be VOC which later turned out to be an osteomyelitis.

Osteomyelitis often occurs in the long bones (the case of our patient in whom the left proximal tibia was affected) in which there is slow vascular flow within the looped vessels of the metaphysic. This combined with microtrauma (ischemia following VOC), is believed to encourage seeding of infection during bacteraemia. Inflammation follows thus, and if purulent material develops, the pressure effect of the subsequent abscess will lead progressively to bone destruction [6]. In osteomyelitis, the primary site of infection is the bone and bone marrow. Any part of the bone may be involved but there is preferential targeting of metaphyseal regions of long bones adjacent to joints [7], the case in our patient was typical of this. Like this case, the usual means of infection is by the haematogenous spread through the bone marrow to the cortex, although direct introduction may complicate trauma or orthopaedic surgery, or may arise from radiation therapy [7] [8]. When pus accumulates, intramedullary pressure increases, resulting in vascular collapse, venous stasis, and further ischemia. Pus accumulating underneath the periosteum elevates it from the cortex, thus further reduces the vascular supply. As this continues to accumulate, the periosteum is breached, followed by the development of mucosal or cutaneous abscesses and fistulae [8]. In cases of untreated or chronic infection, this new bone or involucrum may surround the dead bone, the sequestrum, leading to a "bone-within-bone" appearance. Infection-controlled surgery and being bed-ridden for seven days affirms hospitalization duration according to the classification system whereas patients with advanced osteomyelitis (type B4) are noted for extended hospitalization (months) and high risk of repeated infections and surgery [6].

Mindful of the inadequate diagnostic imaging modalities like bone scintigraphy such as in the present case, diagnosing osteomyelitis in children with SCD can be extremely difficult as they often present with fever and a painful, swollen, tender limb with limited range of motion. These signs and symptoms are similar to those found in patients with a VOC. There was no definitive features on history, physical examination, laboratory testing, or radiological study that could reliably differentiate between osteomyelitis and VOC in our patient, with the possible exception of a positive bacterial culture from the bone [9]. However, a bone biopsy or aspiration is often not performed because it is an invasive procedure and should be done before starting antibiotics to maximize the chances of obtaining a positive culture result [9]. Furthermore, positive bone biopsy cultures such as in this case are reported in only $30 \%$ to $86 \%$ of cases [10], implying that there is still a high false-negative rate. Negative cultures from the peripheral blood such as in this case are even less specific in supporting a diagnosis of osteomyelitis, and they are reported to demonstrate an organism in $30 \%$ to $76 \%$ of cases [10]. Thus, even though the patient was confirmed to have subacute osteomyelitis, there are many cases in which no definitive diagnosis can be made 
as osteomyelitis may show similar clinical and para clinical presentation to VOC in the acute stage. The patient, in tandem with pain in the bone and fever, was initially treated empirically for possible osteo-articular infection or sepsis with broad spectrum antibiotics. These antibiotics were discontinued 48 hours later as the blood culture returned negative. However, in the case of the patient, fever and pain were persistent despite negative blood culture. Clinicians are often faced with the dilemma of whether to treat for osteomyelitis or not [9]; we nevertheless continued antibiotics on this basis and that of a positive urine culture. Failure to treat an unconfirmed case of osteomyelitis can have serious consequences, including weight loss, growth retardation chronic bone damage, persistent sepsis [3], chronic limb deformity and stiffness as well as social stigmatization [9]. However, unnecessary treatment for what is mistakenly thought to be osteomyelitis can have significant psychosocial, financial and healthcare resource implications and can contribute to antibiotic resistance. Patients may also unnecessarily experience adverse effects of the antibiotics and are at risk of complications from central or peripheral intravenous lines that may be inserted to facilitate administration of antibiotics [10].

It is important to highlight that osteomyelitis is much less common than VOC. Studies suggest that VOC is 50 times more common than osteomyelitis in a patient with SCD [11] [12]; as in the onset of symptoms of this patient, she was managed for painful bone crisis which later turned out to be an osteomyelitis. Therefore, even if a patient with SCD presents with fever and bony pain and has risk factors for osteomyelitis, this should be considered in the context of the rarity of osteomyelitis compared with that of VOC. The presence of some or all of the risk factors should nevertheless increase the index of suspicion for osteomyelitis and make the physician more inclined to request imaging or a definitive bone aspirate for culture.

\section{Conclusion}

Differentiating VOC from osteomyelitis is a medically bent tree to straighten as there is no clear-cut clinical difference at an initial stage. These areas biomarkers and/or blood workup and imaging show similar abnormalities in the acute stages. Osteomyelitis, a difficult and dreadful disease of childhood when associated with sickle cell disease and its complications can be severe and even deathly, especially in low income countries. Therefore, there should always be a high index of suspicion of osteomyelitis in all sickle cell patients who present with a painful bone crisis. The clinician should never hesitate to seek more advanced imaging techniques or perform repeated radiographies in order to make an early diagnosis and initiate timely adequate treatment.

\section{Conflicts of Interest}

The authors declare no conflicts of interest regarding the publication of this paper. 


\section{References}

[1] Sap Ngo Um, S., et al. (2019) A Cross Sectional Study of Growth of Children with Sickle Cell Disease, Aged 2 to 5 Years in Yaoundé, Cameroon. The Pan African Medical Journal, 34, 13. https://doi.org/10.11604/pamj.2019.34.85.16432

[2] Ankit, M., et al. (2020) StatPearls-Sickle Cell Anemia. https://www.ncbi.nlm.nih.gov/books/NBK482164/\#_ncbi_dlg_citbx_NBK482164

[3] Fontalis, A., et al. (2019) The Challenge of Differentiating Vaso-Occlusive Crises from Osteomyelitis in Children with Sickle Cell Disease and Bone Pain: A 15-Year Retrospective Review. Journal of Children's Orthopaedics, 13, 33-39. https://doi.org/10.1302/1863-2548.12.180094

[4] AlDallal, S.M. (2017) Osteomyelitis: A Manifestation of Sickle Cell Anaemia. Clinical and Medical Investigation, 2,1-3. https://doi.org/10.15761/CMI.1000132

[5] Mark, W., et al. (1998) Etiology of Osteomyelitis Complicating Sickle Cell Disease. Pediatrics, 101, 296-297. https://doi.org/10.1542/peds.101.2.296

[6] Walker, B.R., Colledge, N.R., Ralston, S.H. and Penman, I.D. (2014) Davidson's Principles and Practice of Medicine. Churchil Livingstone Elsevier, London, 1095.

[7] Kamakshi, S.S., Naik, V., Vittal, K. and Shriyanka, R. (2016) Chronic Suppurative Osteomyelitis: A Case Report. Journal of Advanced Clinical and Research Insights, 3, 220-223. https://doi.org/10.15713/ins.jcri.143

[8] Williams, N.S., Bulstrode, C.J.K. and O’Connel, R. (2013) Bailey and Love's Short Practice of Surgery. CRC Press, New York, 571.

[9] Berger, E., Saunders, N., Wang, L. and Friedman, J.N. (2009) Sickle Cell Disease: Differentiating Osteomyelitis from Vaso-Occlusive Crisis. Archives of Pediatrics and Adolescent Medicine, 163, 251-255. https://doi.org/10.1001/archpediatrics.2008.545

[10] Keeley, K. and Buchanan, G.R. (1982) Acute Infarction of Long Bones in Children with Sickle Cell Anemia. The Journal of Pediatrics, 101, 170-175. https://doi.org/10.1016/S0022-3476(82)80111-X

[11] Buchanan, G.R. (1996) Differentiation of Bone Infarct from Infection in a Child in Sickle Cell Disease. The Pediatric Infectious Disease Journal, 15, 724-725. https://doi.org/10.1097/00006454-199608000-00028

[12] Rudolph, C.D. (2003) Rudolph’s Pediatrics. McGraw-Hill, New York, 904-906. 